\title{
Safety of tumor necrosis factor inhibitor use in patients with concomitant malignancy
}

\author{
Hiep Phan ${ }^{1,2}$, Rick A. Weideman ${ }^{3}$, Daisha J. Cipher ${ }^{4}$, Linda A. Feagins ${ }^{5}$ \\ ${ }^{1}$ Department of Medicine, VA North Texas Healthcare System, Dallas, TX; ${ }^{2}$ Department of Medicine, University of Texas Southwestern Medical \\ Center, Dallas, TX; ${ }^{3}$ Department of Pharmacy, VA North Texas Healthcare System, Dallas, TX; ${ }^{4}$ College of Nursing and Health Innovation, \\ University of Texas at Arlington, Arlington, TX; ${ }^{5}$ Division of Gastroenterology, The University of Texas at Austin Dell Medical School, Austin, TX, \\ $U S A$
}

Background/Aims: Safety for tumor necrosis factor inhibitors (TNFi) in cancer has been focused on risk of incident malignancies, but studies on prognostic effects have been scarce. We determined survival and recurrence rates at 1, 2, and 5 years after cancer diagnosis in patients with and without concurrent TNFi use. Methods: Chart reviews were performed between 1996 and 2015 at the VA North Texas Healthcare System. Cases were patients with inflammatory disease, concomitant malignancy, and TNFi use while controls were patients with inflammatory disease, concomitant malignancy but no TNFi use. Cases and controls were matched for type of malignancy. Analysis was performed with log-rank tests on Kaplan-Meier curves. Results: Thirty-six cases and 72 controls were identified. For cases, survival at 1, 2, and 5 years were 32 (89\%), 31 (86\%), and 29 (81\%) compared to $63(90 \%), 61(87 \%)$, and $51(73 \%)$ for the control group $(P=0.985)$. For cases, recurrence rates at 1,2 , and 5 years were $3(8 \%), 5(14 \%)$, and $6(17 \%)$ compared to $2(3 \%), 5(7 \%)$, and $7(10 \%)$ for the control group $(P=0.158)$. Conclusions: Our findings suggest TNFi may be safely used in select inflammatory disease patients with concurrent cancer if therapy is needed for proper disease control. However, case-by-case consideration in conjunction with an oncologist is recommended while considering the apparent safety of TNFi for patients suffering from active inflammatory diseases despite having a concomitant malignancy. (Intest Res 2020;18:282-288)

Key Words: Tumor necrosis factor inhibitor; Neoplasms; Inflammatory bowel disease; Arthritis, rheumatoid

\section{INTRODUCTION}

Tumor necrosis factor inhibitors (TNFi) are usually considered contraindicated in patients with a recent history of malignancy. General conservative recommendations have included often waiting 5 years after a cancer diagnosis before initiating TNFi therapy. ${ }^{1,2}$ However, these recommendations were based more on expert opinion than supportive data in patients with inflammatory disease.

Received November 5, 2019. Revised December 20, 2019

Accepted December 22, 2019.

Correspondence to Linda A. Feagins, Division of Gastroenterology, The

University of Texas at Austin Dell Medical School, Health Discovery Building, Z0900 1601 Trinity Street, Building B, Austin, TX 78712, USA. Tel: +1-512-

495-5139, Fax: +1-512-495-5839, E-mail: Linda.Feagins@austin.utexas.edu
Initial concerns of increased cancer risk associated with TNFi use arose from data in transplant patients with a history of a previous malignancy. Subsequently, an Food and Drug Administration (FDA) postmarketing report observed 2 out of 26 cases of lymphoma regressing once TNFi therapies were halted in rheumatoid arthritis (RA) and IBD patients. ${ }^{4}$ In addition, a 2006 meta-analysis reported a 3-fold increased risk for all malignancies (pooled OR of 3.3) in patients treated with TNFi therapies. ${ }^{5}$ These data led to an FDA black box warning for TNFi in 2009 in regards to malignancy. ${ }^{4}$ However, when these drugs are stopped in the setting of malignancy, clinicians are often left to deal with patients feeling poorly from active inflammatory disease.

Faced with this difficult clinical situation, various studies have attempted to address this concern by focusing on risk of 
cancer recurrence in patients exposed to TNFi. A retrospective study using Medicare databases found no increased risk of recurrence of breast cancer in patients on immunosuppressants including methotrexate and TNFi. ${ }^{6}$ However, the study excluded cases where TNFi was used during the first year following cancer diagnosis, which leaves the question of continuing TNFi in these patients unanswered. Similarly, a meta-analysis of observational studies also found no increase in cancer recurrence rates but the majority of these studies also excluded cases with TNFi use in the immediate period following cancer diagnosis. ${ }^{7}$ In contrast, survival in cancer patients with concurrent TNFi use has been less well studied. Therefore, our primary aim was to assess the survival outcomes of patients with TNFi exposure leading up to or during concomitant cancer diagnosis and treatment. Our secondary objective was to assess recurrence rates for patients with malignancy exposed to TNFi use.

\section{METHODS}

\section{Study Design}

This study was a retrospective chart review performed for the period between January 1, 1996 and November 30, 2015 at the VA North Texas Healthcare System. Both the cases and control group were followed from the time of malignancy diagnosis until the end of study (EOS) date, which was designated as patient's death, no further follow-up available or November 30, 2015. Inflammatory diseases included were CD, UC, RA, ankylosing spondylitis, spondyloarthropathy (SpA), psoriasis and psoriatic arthritis (PsA). Malignancies included were all hematologic and solid tumors with the exclusion of non-melanoma skin cancers.

\section{Patient Selection and Matching}

Potential cases were identified and cross referenced across multiple databases including local VA pharmacy databases for TNFi exposure (infliximab, adalimumab, certolizumab pegol, golimumab, or etanercept), ICD-9 and ICD-10 codes in the Veterans Health Information Systems and Technology Architecture system and VA pathology Systemized Nomenclature of Medicine (SNOWMED) database. Cases were patients who developed malignancy and were exposed to a TNFi 2 years prior to developing or within 5 years after developing a malignancy with a minimum consecutive exposure length of 3 months. No maximum exposure length was specified. Cases were manually reviewed for the inclusion criteria. In order to reduce the bias on survival by malignancy type, each case was matched for malignancy type to 2 randomly chosen (using a random number generator) control patients without TNF exposure from a pool of potential TNFi naïve controls. These were identified by cross referencing data from the SNOWMED pathology database, the Dallas VA IBD database, and an ICD-9 and ICD-10 search for rheumatologic diseases and psoriasis. The inclusion criteria for the control group was confirmed inflammatory disease and malignancy diagnosis without TNFi exposure ever. The preliminary control group was manually reviewed for inclusion criteria prior to matching.

\section{Data Collection}

Cases meeting inclusion criteria had their charts reviewed for demographics, malignancy survival, inflammatory disease profiles, inflammatory disease medication history and comorbid conditions at the 1-, 2-, 5-year and EOS timepoints. Malignancy diagnosis, staging, treatment, recurrence and survival was determined by review of pathology, medical and surgical oncology notes. Severity and treatment of inflammatory diseases was assessed using clinic notes and pharmacy records at the time of cancer diagnosis and followed through all possible timepoints. Comorbid conditions were assessed for each patient using the Charlson Comorbidity Index (CCI) at each of the study timepoints. Patients in the control group were reviewed for the same data points.

\section{SEER Comparison}

Survival rates were further assessed in the TNFi exposed group by comparing them at the previously defined timepoints to the National Cancer Institute's Surveillance, Epidemiology and End Results (SEER) program database. Patients were matched to the SEER Relative Survival by Year of Diagnosis data on the basis of gender, cancer type, year of diagnosis, and length of follow-up. The matching relative survival rates from the SEER database were averaged for the 1-, 2-, and 5-year timepoints to reflect the surviving TNFi exposed patients at these timepoints.

\section{Statistical Analysis}

Continuous variables were analyzed with Mann-Whitney tests or independent samples $t$-tests, and categorical variables were analyzed with Fisher exact tests. Kaplan-Meier survival curves were compared with log-rank tests. The study alpha was set to 0.05. Analyses were performed with GraphPad (Instat, 2009; GraphPad Software Inc., San Diego, CA, USA) and SPSS 23.0 (IBM Corp., Armonk, NY, USA).

Survival time was calculated from the date of cancer diag- 
nosis to last date of follow-up, death date or November 30, 2015, whichever occurred first. Survival following cancer diagnosis in the 2 matched groups was depicted using KaplanMeier curves. Recurrence time was calculated from the date of cancer diagnosis to the first date of recurrence. Cancer recurrence time between the 2 groups were also depicted with Kaplan-Meier curves counting death as censored.

Table 1. Baseline Demographics of Veterans with Inflammatory Disease Comparing Patients Exposed or Not Exposed to TNFi

\begin{tabular}{|c|c|c|c|}
\hline Variable & TNFi exposed $(n=36)$ & TNFi naïve $(n=72)$ & $P$-value \\
\hline Sex & & & 1.00 \\
\hline Male & $35(97)$ & $69(96)$ & \\
\hline Female & $1(3)$ & $3(4)$ & \\
\hline \multicolumn{4}{|l|}{ Ethnicity } \\
\hline Caucasian & $34(94)$ & $53(74)$ & $0.01^{\mathrm{a}}$ \\
\hline African American & $1(3)$ & $16(22)$ & $0.01^{\mathrm{a}}$ \\
\hline Hispanic & $1(3)$ & $2(3)$ & 1.00 \\
\hline Native American & 0 & $1(1)$ & 1.00 \\
\hline Charlson Comorbidity Index & $2.5 \pm 1.7$ & $2.1 \pm 1.9$ & 0.19 \\
\hline \multicolumn{4}{|l|}{ Smoking status } \\
\hline Never & $7(19)$ & $6(8)$ & 0.12 \\
\hline Former & $18(50)$ & $31(43)$ & 0.54 \\
\hline Active & $11(31)$ & 35 (49) & 0.10 \\
\hline \multicolumn{4}{|l|}{ Inflammatory disease type } \\
\hline \multicolumn{4}{|l|}{ IBD only } \\
\hline$C D$ & $1(3)$ & $2(3)$ & 1.00 \\
\hline UC & $2(6)$ & $4(6)$ & 1.00 \\
\hline Rheumatoid arthritis only & $22(61)$ & $48(67)$ & 0.67 \\
\hline Psoriasis only & $3(8)$ & $10(13)$ & 0.54 \\
\hline \multicolumn{4}{|l|}{ Spondyloarthropathy only } \\
\hline Ankylosing spondylitis only & $2(6)$ & $2(3)$ & 0.60 \\
\hline IBD+spondyloarthropathy & $1(3)$ & 0 & 0.33 \\
\hline Psoriasis+psoriatic arthritis & $5(14)$ & $6(8)$ & 0.50 \\
\hline Age at inflammatory disease diagnosis (yr) & $46.8 \pm 12.8$ & $52.7 \pm 15.0$ & $0.02^{\mathrm{a}}$ \\
\hline Inflammatory disease duration (yr) & $22.3 \pm 11.0$ & $19.1 \pm 14.5$ & $0.04^{\mathrm{a}}$ \\
\hline \multicolumn{4}{|c|}{ Inflammatory disease medication profile at cancer diagnosis } \\
\hline \multicolumn{4}{|l|}{ Concomitant medications } \\
\hline Oral steroids & $4(11)$ & $11(15)$ & 0.77 \\
\hline Topical steroids & $14(39)$ & $20(28)$ & 0.28 \\
\hline 5-Aminosalicylates & $3(8)$ & $13(18)$ & 0.25 \\
\hline Immunomodulators (thiopurines, methotrexate) & $16(44)$ & $25(35)$ & 0.40 \\
\hline TNFi & $29(81)$ & 0 & - \\
\hline Disease-modifying antirheumatic drugs & 7 (19) & $15(21)$ & 1.00 \\
\hline Psoriatic therapy & $5(14)$ & $5(7)$ & 0.30 \\
\hline
\end{tabular}

Values are presented as number (\%) or mean \pm SD.

a Statistically significant, $P<0.05$.

TNFi, TNF inhibitors. 


\section{Ethical Considerations}

This study was approved by the Institutional Review Board at the VA North Texas Healthcare System (IRB No.\#17-038). This study is a retrospective study using medical record review and so informed consent was waived.

\section{RESULTS}

\section{Baseline Demographics, Cancer Stage and Treatment}

A total of 1,433 patients were identified as ever having received TNFi at the VA North Texas Healthcare System within the study period. Of those, 36 were identified with concomitant malignancy and met the inclusion criteria. For the control cohort, 357 potential patients were identified with inflammatory disease and malignancy meeting the inclusion criteria. Of those, 72 were matched to the cases based on malignancy type. All subsequent analyses were restricted to these patients.

The 36 cases with inflammatory disease, concomitant malignancy, and TNFi use comprised 3 patients with IBD, 22 with RA, 5 with PsA, 2 with SpA, 1 with both IBD and SpA, and 3 with psoriasis. The 72 controls with inflammatory disease, concomitant malignancy, but no TNFi use included 6 with IBD, 48 with RA, 10 with psoriasis, 6 with PsA, and 2 with SpA. Malignancies diagnosed included 30 prostate adenocarcinomas, 18 head \& neck cancers, 15 lung cancers ( 5 adenocarcinomas, 9 squamous cell carcinomas, 1 small cell carcinoma), 15 malignant melanomas, 12 kidney cancers (11 renal cell carcinomas and 1 transitional cell carcinoma), 9 hematologic malignancies ( 2 plasma cell myelomas, 5 malignant lymphoma, 1 multiple myeloma, 1 chronic lymphoid leukemia), 3 colon adenocarcinomas, 3 breast ductal carcinomas, and 3 urinary bladder transitional cell carcinomas.

Cases and controls were predominantly white males. However, interestingly, there were notable differences in the ethnic composition of the 2 groups. The cases had significantly fewer African Americans than the control group ( $3 \%$ vs. $22 \%, P=0.01$ ). Otherwise, the groups were similar regarding type of inflammatory disease, CCI, tobacco use, or concomitant medication use at the time of cancer diagnosis (Table 1). There was no significant difference in composition of inflammatory diseases between the groups; with RA comprising most patients. However, the cases were diagnosed on average 6 years younger and had slightly longer disease duration than the control group.

Cancer staging at diagnosis was similar between cases and controls (Table 2), with more than $50 \%$ of patients with stage I or II disease. Moreover, choices of cancer treatments (chemotherapy, radiation, surgery and/or hormonal) were similar between the 2 groups.

Table 2. Malignancy Types, Stage at Diagnosis, and Treatments for Patients Exposed or Not Exposed to TNFi

\begin{tabular}{|c|c|c|c|}
\hline Variable & $\begin{array}{c}\text { TNFi } \\
\text { exposed } \\
(n=36)\end{array}$ & $\begin{array}{l}\text { TNFi naïve } \\
(n=72)\end{array}$ & $P$-value \\
\hline \multicolumn{4}{|l|}{ Cancer types } \\
\hline Breast & $1(3)$ & $2(3)$ & 1.00 \\
\hline Colon & $1(3)$ & $2(3)$ & 1.00 \\
\hline Head \& neck & $6(17)$ & $12(17)$ & 1.00 \\
\hline Hematologic & $3(8)$ & $6(8)$ & 1.00 \\
\hline Lung & $5(14)$ & $10(14)$ & 1.00 \\
\hline Prostate & $10(28)$ & $20(28)$ & 1.00 \\
\hline Renal & $4(11)$ & $8(11)$ & 1.00 \\
\hline Skin & $5(14)$ & $10(14)$ & 1.00 \\
\hline Urinary bladder & $1(3)$ & $2(3)$ & 1.00 \\
\hline Age of malignancy diagnosis (yr) & $62.8 \pm 8.9$ & $65.4 \pm 8.6$ & 0.15 \\
\hline Months of follow-up & $64.7 \pm 54.8$ & $62.7 \pm 46.8$ & 0.95 \\
\hline \multicolumn{4}{|l|}{ Stage of cancer diagnosis } \\
\hline In situ & $6(17)$ & $12(17)$ & 1.00 \\
\hline Stage 1 & $17(47)$ & $24(33)$ & 0.21 \\
\hline Stage 2 & $5(14)$ & $19(26)$ & 0.22 \\
\hline Stage 3 & $2(5)$ & $6(8)$ & 0.72 \\
\hline Stage 4 & $6(17)$ & $11(15)$ & 1.00 \\
\hline \multicolumn{4}{|l|}{ Cancer treatments } \\
\hline Chemotherapy & $4(11)$ & $13(18)$ & 0.41 \\
\hline Hormonal & $2(6)$ & $3(4)$ & 1.00 \\
\hline Radiation & $8(22)$ & $18(25)$ & 0.82 \\
\hline Surgery & $25(69)$ & $46(64)$ & 0.67 \\
\hline Months of TNFi exposure & & & - \\
\hline Before cancer diagnosis & $32.8 \pm 33.8$ & 0 & \\
\hline After cancer diagnosis & $23.1 \pm 35.6$ & 0 & \\
\hline If TNFi stopped, TNFi restarted? & & & - \\
\hline 1 Year & $11(31)$ & 0 & \\
\hline 2 Years & $1(3)$ & 0 & \\
\hline 5 Years & 0 & 0 & \\
\hline End of study & 0 & 0 & \\
\hline
\end{tabular}

Values are presented as number (\%) or mean \pm SD. Cases matched 1:2 with controls for malignancy type.

TNFi, TNF inhibitors. 


\section{TNFi Use}

For cases, 28 (77.8\%) were on TNFi before malignancy diagnosis with an average of 32.8 months of TNFi exposure. Of those, 23 (82.8\%) had their TNFi reflexively held at the time of malignancy diagnosis. The other 5 (17.2\%) continued their TNFi through the cancer diagnosis and treatment period. Overall, there were 17 (47.2\%) who started (or restarted) TNFi therapy within 5 years of the malignancy diagnosis including 5 patients restarting on TNFi within the same year as their cancer diagnosis. The average TNFi exposure after cancer diagnosis was 23.1 months. Interestingly, the cases had significantly more patients on systemic steroids at the 1-year (53\% vs. 17\%), 2-year (42\% vs. $11 \%$ ) and 5 -year (31\% vs. 3\%) timepoints post cancer diagnosis as compared to the controls.

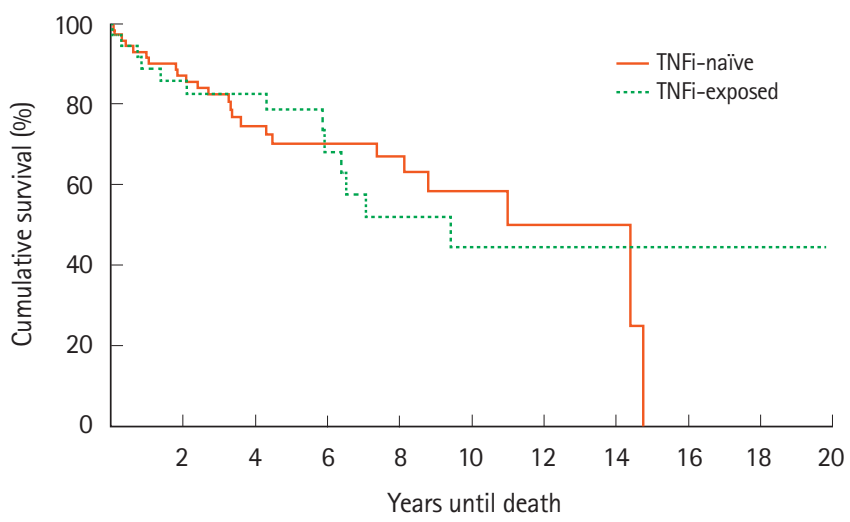

Fig. 1. Survival after cancer diagnosis compared between patients exposed or not exposed to TNF inhibitors (TNFi). The probability of accelerated mortality did not significantly differ between the TNFi exposed and TNFi naïve groups. $\chi^{2}(1)=0.0, P=0.985$

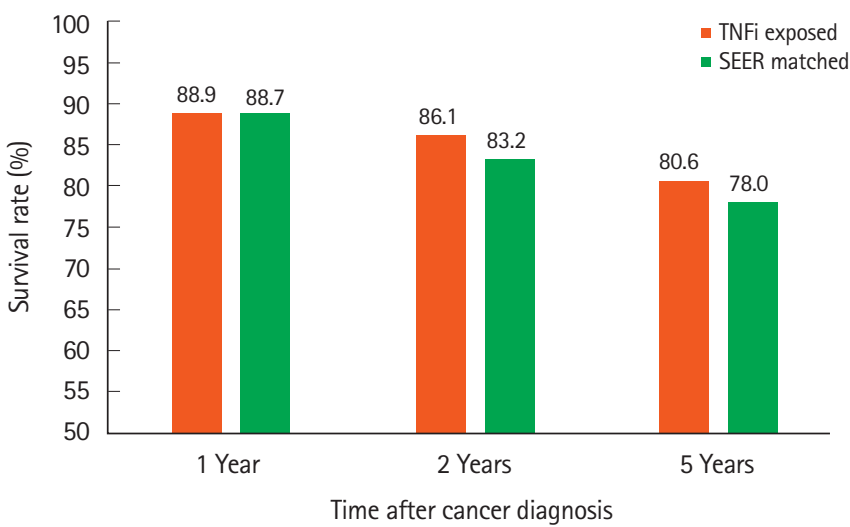

Fig. 2. Survival rates for cases (TNFi exposed) compared with the National Cancer Institute's Surveillance, Epidemiology and End Results (SEER) national data matched for gender, cancer type and diagnosis year. TNFi, TNF inhibitors.

\section{Cancer Survival and Recurrence Rates}

For cases, survival at 1, 2, 5 years and at EOS follow-ups were 32 (89\%), 31 (86\%), 29 (81\%), and 24 (64\%), respectively compared to 63 (90\%), 61 (87\%), 51 (73\%), and 45 (64\%) for the control group (Fig. 1). Kaplan-Meier analysis indicated that there was no significant difference between cases and controls in survival $\left(\chi^{2}(1)=0.0, P=0.985\right)$. Similar results were found upon comparison of the cases survival rates to the SEER data (Fig. 2). Survival at 1 year was $88.9 \%$ vs. $88.7 \%$, $86.1 \%$ vs. $83.2 \%$ at 2 years, and $80.6 \%$ vs. $78.0 \%$ at 5 years (cases vs. SEER matched).

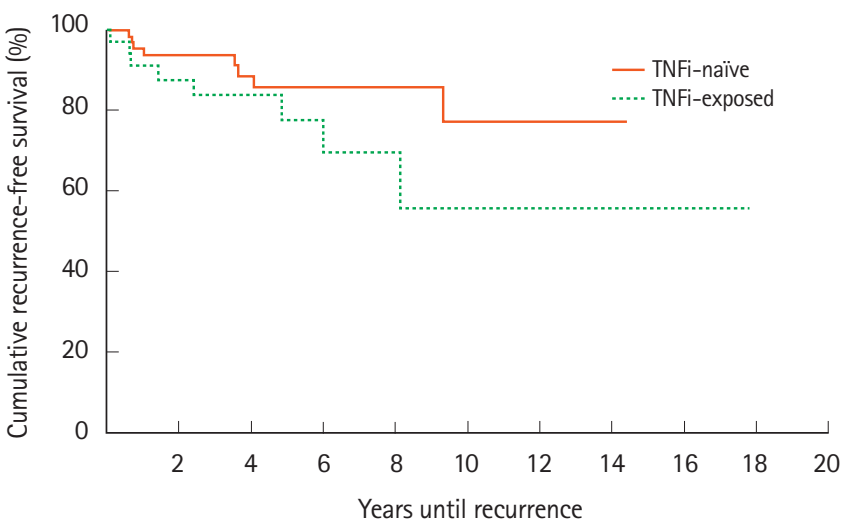

Fig. 3. Cancer recurrence rates after initial cancer diagnosis compared between patients exposed or not exposed to TNF inhibitors (TNFi). The probability of accelerated recurrence also did not significantly differ between the TNFi exposed and TNFi naïve groups. $\chi^{2}(1)=2.0, P=0.158$.

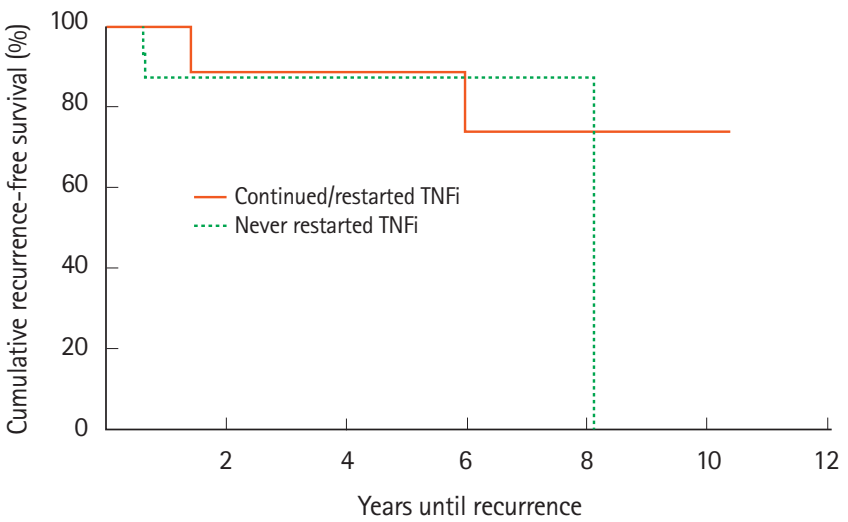

Fig. 4. Cancer recurrence rates after initial cancer diagnosis compared between patients treated with TNF inhibitors (TNFi) that was discontinued at cancer diagnosis and not restarted compared to patients who continued or restarted TNFi soon after cancer diagnosis. Kaplan-Meier analysis indicated that there was no significant difference between the cases and controls in accelerated recurrence. $\chi^{2}(1)=0.57, P=0.45$. 
For cases, recurrence rates at 1, 2, 5 years and at EOS followups were $3(8 \%), 5$ (14\%), 6 (17\%), and 8 (22\%), respectively compared to 2 (3\%), 5 (7\%), 7 (10\%), and 9 (13\%) for the control group (Fig. 3). Kaplan-Meier analysis indicated that there was no significant difference between the cases and controls in accelerated recurrence $\left(\chi^{2}(1)=2.0, P=0.158\right)$.

On subgroup analysis, we compared cancer recurrence rates between patients who were exposed to TNFi and stopped it at cancer diagnosis $(\mathrm{n}=18)$ to patients who continued TNFi through cancer treatment or restarted TNFi soon after cancer diagnosis $(n=10)$ (Fig. 4). There was no statistically significant difference in accelerated recurrence between these 2 groups $\left(\chi^{2}(1)=0.57, P=0.45\right)$.

\section{DISCUSSION}

In our matched cohorts of veterans with inflammatory disease and malignancies, we found that the use of TNFi did not affect overall survival or cancer recurrence. Similarly, when our veteran cohort treated with TNFi was compared with age, sex and cancer type matched control patients from the SEER database, there was no difference in overall survival. These findings are in line with recent studies in both RA and IBD populations. A Swedish cohort study specifically evaluating RA patients with breast cancer who were treated with TNFi found no differences in recurrence rates with similar patients who did not receive treatment with $\mathrm{TNFi}^{8}{ }^{8}$ However, the median time to starting TNFi after breast cancer diagnosis in this study was over 9 years later and thus not generalizable to patients with a recent diagnosis of cancer who need TNFi. Similarly, a study using a British registry of RA patients starting biologics reported no difference in incident malignancies of all types in patients using TNFi as compared to those treated with traditional disease-modifying antirheumatic drugs, but the average time between starting a TNFi and prior cancer diagnosis was 11.5 years. ${ }^{9}$ A strength of our study was that we excluded patients starting TNFi 5 years or more after malignancy diagnosis, improving generalizable to patients needing TNFi at the time of their cancer diagnosis or soon thereafter.

A French study identified a cohort of 79 patients with IBD who received TNFi on average 17 months after diagnosis with malignancy. They found that 1-year survival was $96 \%$ and 5-year survival was $66 \%$ with a total of $19 \%$ developing incident cancers. ${ }^{10}$ Unfortunately this study had no control group with which to compare outcomes. A retrospective study from New York including 106 patients with IBD who were treated with TNFi after a malignancy reported that there was no difference in incident cancer rates for those treated with TNFi alone, combination TNFi and immunomodulator or controls. ${ }^{11}$ However, there were significant baseline differences between the TNFi treated patients in this study and the controls including differences in cancer staging and cancer types which may bias survival heavily. Another strength of our study is that we included a control group without TNFi use who also had a history of malignancy and were matched 2:1 to the cases by type of cancer. Our cases and controls were similar in regard to inflammatory disease type, CCI, smoking status, concomitant medications at the time of cancer diagnosis, cancer type, staging and cancer treatments. Our groups only differed in the TNFi group having fewer African Americans than the control group as well as the TNFi treated group having a slightly longer disease duration (on average 3 years longer) and being diagnosed slightly younger with their inflammatory disease (on average 6 years younger). In addition to our control cohort of veterans, we additionally included an age, sex, and cancer-matched control survival rates from the SEER database, further supporting our conclusions.

Our data included detailed chart review that allowed us to calculate not only survival rates after cancer diagnosis, but to also assess recurrence rates of the original cancer that was diagnosed. In our Kaplan-Meier analysis, while there was a numerically higher recurrence rate in the patients treated with $\mathrm{TNFi}$, there was no statistical significant difference between those treated or not treated with TNFi. Interestingly, when we analyzed the data in subgroups, comparing recurrence rates between those who were exposed to TNFi and stopped it at cancer diagnosis with those who continued it or restarted it soon after cancer diagnosis, there was again no significant difference in recurrence rates $(P=0.45)$. Our study is the first to compare these 2 strategies of TNFi use in the time around a malignancy diagnosis; stopping TNFi without restarting versus continuing or restarting soon after cancer diagnosis.

Despite its many strengths, several limitations should be taken into consideration for this study. These include the relatively small sample size and a heterogeneous population of inflammatory diseases. This limits the analysis of individual cancer types and individual inflammatory diseases. Additionally, our subgroup analysis of the discontinuation of TNFi as compared to continuing or restarting soon after cancer diagnosis is likely underpowered given the small sample size. Further, our veteran population is predominately male making it less generalizable to women. Lastly, despite the well-matched 
cohorts, given the retrospective nature of the study, there is always the potential for selection bias of unmeasured characteristics that may have influenced providers to discontinue versus restart TNFi after cancer diagnosis.

In conclusion, the findings of this study suggest TNFi appear safe to use in selected inflammatory patients with concomitant cancer. However, clinical decisions to start TNFi in these patients should still be made on a case-by-case basis in conjunction with oncologists by taking the apparent safety of the TNFi into consideration for patients suffering from active inflammatory diseases despite having a concomitant malignancy. Future studies should include multiple institutions to provide larger cohorts of patients to allow disease specific and cancer specific analysis of outcomes. Moreover, if feasible, a randomized study of stopping therapy versus continuing through cancer treatment would bring even stronger data to guide our clinical practice.

\section{FINANCIAL SUPPORT}

The authors received no financial support for the research, authorship, and/or publication of this article.

\section{CONFLICT OF INTEREST}

Feagins LA has previously participated in research with Takeda, Inc. All other authors have no potential conflicts.

\section{AUTHOR CONTRIBUTION}

Conceptualization: Feagins LA. Methodology: Feagins LA, Phan H, Weideman RA. Formal analysis: Feagins LA, Phan H, Cipher DJ. Writing - original draft: Phan H. Writing - review and editing: Feagins LA. Final approval: all authors.

\section{ORCID}

Phan $\mathrm{H}$ https://orcid.org/0000-0002-0778-0826

Weideman RA https://orcid.org/0000-0002-8129-6557

Cipher DJ

Feagins LA https://orcid.org/0000-0001-7022-9538 https://orcid.org/0000-0001-7758-3257

\section{REFERENCES}

1. Elandt K, Aletaha D. Treating rheumatic patients with a malignancy. Arthritis Res Ther 2011;13:223.
2. Nguyen GC. First do no harm: is it safe to use immunosuppressants in inflammatory bowel disease patients with prior cancer? Gastroenterology 2016;151:22-24.

3. Penn I. Evaluation of transplant candidates with pre-existing malignancies. Ann Transplant 1997;2:14-17.

4. Center for Drug Evaluation and Research. Drug Safety Information for Heathcare Professionals - Questions and Answers - TNF Blockers 8/25/2009. https://www.fda.gov/drugs/postmarket-drug-safety-information-patients-and-providers/information-tumor-necrosis-factor-tnf-blockers-marketed-remicade-enbrel-humira-cimzia-and-simponi.

5. Bongartz T, Sutton AJ, Sweeting MJ, Buchan I, Matteson EL, Montori V. Anti-TNF antibody therapy in rheumatoid arthritis and the risk of serious infections and malignancies: systematic review and meta-analysis of rare harmful effects in randomized controlled trials. JAMA 2006;295:2275-2285.

6. Mamtani R, Clark AS, Scott FI, et al. Association between breast cancer recurrence and immunosuppression in rheumatoid arthritis and inflammatory bowel disease: a cohort study. Arthritis Rheumatol 2016;68:2403-2411.

7. Micic D, Komaki Y, Alavanja A, Rubin DT, Sakuraba A. Risk of cancer recurrence among individuals exposed to antitumor necrosis factor therapy: a systematic review and meta-analysis of observational studies. J Clin Gastroenterol 2019;53:el-el1.

8. Raaschou P, Simard JF, Neovius M, Askling J; Anti-Rheumatic Therapy in Sweden Study Group. Does cancer that occurs during or after anti-tumor necrosis factor therapy have a worse prognosis? A national assessment of overall and site-specific cancer survival in rheumatoid arthritis patients treated with biologic agents. Arthritis Rheum 2011;63:1812-1822.

9. Silva-Fernández L, Lunt M, Kearsley-Fleet L, et al. The incidence of cancer in patients with rheumatoid arthritis and a prior malignancy who receive TNF inhibitors or rituximab: results from the British Society for Rheumatology Biologics Register-Rheumatoid Arthritis. Rheumatology (Oxford) 2016; 55:2033-2039.

10. Poullenot F, Seksik P, Beaugerie L, et al. Risk of incident cancer in inflammatory bowel disease patients starting anti-TNF therapy while having recent malignancy. Inflamm Bowel Dis 2016; 22:1362-1369.

11. Axelrad J, Bernheim O, Colombel JF, et al. Risk of new or recurrent cancer in patients with inflammatory bowel disease and previous cancer exposed to immunosuppressive and anti-tumor necrosis factor agents. Clin Gastroenterol Hepatol 2016;14:58-64. 